\title{
The Trauma-Vascular Surgeon: A Global Necessity
}

\author{
Ernest E Moore MD \\ Ernest E Moore Shock Trauma Center at Denver Health, Denver, Colorado, USA
}

Trauma is the most common cause of death in the United States in the population under 45 years of age. Despite the National Academy of Medicine, Engineering, and Science (NAMES) white paper calling for "Zero Preventable Deaths following Injury" [1], mortality attributed to trauma has increased in the United States over the past decade [2]. Uncontrolled bleeding is the leading cause of preventable death following injury in both the civilian and military settings and, thus, is a conspicuous target to improve outcome. In recent multicenter studies the time to death from bleeding has been reported to be 1.6-2.3 hours, and a third of these fatalities occur within the first hour [3-9]. The Hybrid Operating Room construct provides a tremendous opportunity to accomplish prompt control of life and limb threatening bleeding [10]. However, a major challenge to optimize these resources for trauma is the immediately availability of surgeons in regional trauma centers capable of performing open and endovascular control of bleeding. Similarly, the military needs trauma surgeons with vascular capabilities. Currently there is an emerging crisis to ensure this timely surgical care, but the underlying forces are multifactorial [11]. While I am convinced this is a worldwide problem, my comments will be limited to the US experience where penetrating wounds remain common in urban settings.

One of the major factors in this evolving crisis is the decline in vascular skills of the trauma surgeon. This deterioration begins with general surgery training which is detailed in publicly available national reports. A recent

\section{Corresponding author:}

Ernest E Moore MD, Ernest E Moore Shock Trauma Center at Denver Health, Denver, Colorado, USA.

Email: Ernest.Moore@dhha.org

(c) 2019 CC BY 4.0 - in cooperation with Depts. of Cardiothoracic/ Vascular Surgery, General Surgery and Anesthesia, Örebro University Hospital and Örebro University, Sweden review of the Accreditation Council for Graduate Medical Education (ACGME) case logs during the last 15 years has documented a $38 \%$ decrease in open arterial operations performed by general surgery residents [12]. The reduction in vascular care has been particularly conspicuous over the past decade, with current general surgery residents finishing with less than 2 vascular trauma procedures [13]. This decline has been attributed to the dramatic shift to endovascular therapy, increased vascular surgery trainees $(5+2$ fellowships and $0-5$ vascular programs), as well as the increased number of general surgery training programs. The current Trauma and Acute Care Surgery (TACS) model in the United States has exacerbated this crisis. Somewhat ironically, the TACS concept was formalized to re-establish operative experience for trauma surgeons by integrating emergency general surgery (EGS). Unfortunately, in most trauma centers, this has primarily increased nocturnal experience with laparoscopic appendectomies and cholecystectomies with the virtual elimination of open vascular operations [14]. The TACS shift construct and extensive surgical intensive care unit (SICU) responsibilities have further limited the capacity of the modern TACS surgeon to participate in vascular work. Finally, the unbridled over-designation of trauma centers has reduced the trauma volume at many academic trauma centers, further diluting experience for training and maintaining vascular skills.

On the other side is maintaining trauma expertise in those formally trained in vascular surgery. Most vascular surgeons are understandably reluctant to participate in in-house calls for trauma surgery, and few are interested in maintaining expertise in non-vascular trauma procedures. The limited experience with open thoracic, abdominal, and pelvic procedures is a further dilemma for 5-0 trained vascular surgeons who have focused on endovascular work.

There are a number of potential solutions to the provision of timely vascular care in regional trauma centers, and I would like to focus on training the next generation of trauma-vascular surgeons (TVSs). Following completion of a general surgical residency, the current TACS 
fellowship consists of a year-long ACGME surgical critical care (SCC) fellowship with EGS and on-call trauma duties, and a second year with minimal case criteria for thoracic, vascular, and hepatobiliary procedures as well as additional EGS and trauma calls [15]. Although a shift from rotation-based duties to minimal longitudinal cases is intuitively attractive, the conspicuous downside is ensuring technical competency. I acknowledge that one year focusing on SCC certification is fundamental, but submit that the second year should have the flexibility to enable the option of a year focusing on acquiring open and endovascular skills. This fellowship will be limited to relatively high-volume urban trauma centers in an academic environment supporting this new training paradigm. Currently the Adams Cowley Shock Trauma Center in Baltimore is the best example in the United States.

In summary, I strongly believe EndoVascular Resuscitation and Trauma Management (EVTM) is the optimal international forum to solve the existing international crisis of ensuring the immediate availability of a skilled TVS, 24 hours a day, 7 days a week, 365 days a year.

\section{REFERENCES}

[1] Berwick DM, Downey AS, Cornett EA. A national trauma care system to achieve zero preventable deaths after injury: recommendations from a National Academies of Sciences, Engineering, and Medicine Report. JAMA. 2016;316:927-8.

[2] Rhee P, Joseph B, Pandiit V, et al. Increasing trauma deaths in the United States. Ann Surg. 2014;260:13-21.

[3] Dutton RP, Stansbury LG, Leone S, et al: Trauma mortality in mature trauma systems: are we doing better? An analysis of trauma mortality patterns. J Trauma. 2010; 69:620-6.

[4] Holcomb JH, Tilley BC, Baranuik S, et al. Transfusion of plasma, platelets, and red cells in a $1: 1: 1$ vs a $1: 1: 2$ ratio and mortality in patients with severe trauma: the PROPPR randomized clinical trial. JAMA. 2015; 313:471-82.
[5] Oyeniti BT, Fox EE, Scerbo M, et al. Trends in 1079 trauma deaths at a level 1 trauma center: impact of bleeding control bundle. Injury. 2017;48:5-12.

[6] Moore EE, Moore FA, Fabian TC, et al. Human polymerized hemoglobin for treatment of hemorrhagic shock when blood is unavailable: the USA multicenter trial. J Am Coll Surg. 2009;208:1-13.

[7] Moore HB, Moore EE, Chapman MP, et al. Plasma-first resuscitation to treat haemorrhagic shock during emergent ground transportation in an urban area: a randomized trial. Lancet. 2018;392:283-91.

[8] Sperry JL, Guyette FX, Brown JB, et al: Prehospital plasma during air medical transport in trauma patients at risk for hemorrhagic shock. N Eng J Med. 2018; 26:315-26.

[9] Tisherman SA, Schmicker RH, Brasel K, et al: Detailed description all deaths in both the shock and traumatic brain injury hypertonic saline trials of the Resuscitation Outcomes Consortium. Ann Surg. 2015;261:586-90.

[10] Kirkpatrick AW, Vis C, Dube M, et al. The evolution of a purpose designed hybrid trauma room from the trauma service perspective: The RAPTOR (Resuscitation with Angiography Percutaneous Treatments and Operative Resuscitations). Injury. 2014;45:1413-21.

[11] Rasmussen TE, Woodsen J, Rich NM, Mattox KL. Vascular trauma at a crossroads. J Trauma. 201;70: 1291-3.

[12] Potts JR, Valentine RJ. Declining resident experience in open vascular operations threatens the status of vascular surgery bas an essential content area of general surgery training. Ann Surg. 2018;268:665-73.

[13] Shannon AH, Robinson WF, Hanks JB, Potts JR. Impact of new vascular fellowship programs on vascular surgery operative volume of residents in associated general surgery programs. J Am Coll Surg. 2018;228: 525-33.

[14] Pottenger BC, Galante JM, Wisner DH. The modern acute car surgeon: characterization of an evolving surgical niche. J Trauma Acute Care Surg. 2015;78:120-5.

[15] Davis KA, Dente CJ, Burlew C, et al. Refining the operative curriculum of the acute care surgery fellowship TACS. J Trauma Acute Care Surg. 2015; 78:192-6. 